\title{
REFLEXO PUDENDO-ANAL EM MULHERES NORMAIS
}

\author{
Geraldo de Aguiar Cavalcanti', Gilberto Mastrocola Manzano², Homero Bruschini, \\ Lydia Maria Giuliano ${ }^{4}$, Miguel Srougi $^{5}$, João Antônio Maciel Nóbrega ${ }^{6}$
}

\begin{abstract}
RESUMO - São descritas observações do registro do reflexo pudendo-anal em mulheres sem queixas de incontinência urinária. Foram estudadas 31 voluntárias adultas, com estimulação elétrica bilateral e independente do clitóris e registros de superfície em ambos os lados do músculo esfíncter externo do ânus (EEA). As respostas foram obtidas com pulsos duplos de 0,2 ms de duração e intervalos de $5 \mathrm{~ms}$, aplicados a uma freqüência menor que $0,5 \mathrm{~Hz}$. Foram medidas as latências iniciais das respostas. Não foram evidenciadas diferenças entre as respostas obtidas de cada lado do EEA e nem entre os lados, com relação aos estímulos. Uma das voluntárias não apresentou respostas após estimulação de um dos lados. Não foram observadas diferenças relacionadas a paridade total e nem com a presença de partos vaginais. A idade e o índice de massa corpórea não se correlacionaram com as respostas. Em 12\% das respostas, a medida das latências foi dificultada pela baixa relação sinal-ruído.
\end{abstract}

PALAVRAS-CHAVE: assoalho pélvico, neurofisiologia, urodinâmica, reflexos sacrais, incontinência urinária.

\begin{abstract}
Pudendo-Anal Reflex in Normal Women
ABSTRACT - The pudendo-anal reflex was studied in a sample of 31 normal women. Responses were obtained after bilateral independent stimulation of the clitoris, with surface recordings from both sides of the external anal sphincter. Reponses were elicited with double-pulses of 0,2 ms duration with a interstimulus interval of $5 \mathrm{~ms}$, frequency of stimulation was lower than $0,5 \mathrm{~Hz}$. A minimal of four responses were recorded after supramaximal stimulation. In one volunteer no response was recorded after unilateral stimulation. Latencies of the responses from the right and left sides of the anal sfincter after right and left stimulation were $36.35 \pm 6.37,36.28 \pm 6.23,35.88 \pm 4.68,36.44 \pm 4.45 \mathrm{~ms}$, respectively. No relation was detected between latencies and age, body mass index and parity (considering either total parity or vaginal delivery only). In $12 \%$ of the recordings uncertainty was introduced in the latency measurements related to a poor signal-noise ratio.
\end{abstract}

KEY WORDS: pelvic floor, neurophysiology, urodynamics, urinary incontinence, sacral reflexes.

Várias respostas reflexas podem ser obtidas dos músculos constituintes do assoalho pélvico. Elas podem ser classificadas de acordo com a aferência em somato-somáticas ou viscero-somáticas. Em clínica, estas respostas são usualmente estudadas com a finalidade de se avaliar o cone medular ou as raízes sacrais S2-S41. O estímulo cutâneo na região perineal ou na porção proximal das coxas pode provocar respostas reflexas dos músculos do assoalho pélvico. Dessa forma, contrações do músculo esfíncter externo do ânus podem ser evocadas (clássico reflexo cutâneo-anal) ${ }^{2}$. A estimulação dos nervos cutâneos do pênis ou clitóris desencadeia respostas reflexas que podem ser registradas em vários músculos do assoalho pélvico, dando origem a diferen- tes reflexos dependendo do músculo no qual a resposta foi registrada. Citam-se, como exemplo, o reflexo bulbo-cavernoso, isquio-cavernoso e pudendo-anal ${ }^{1}$. Embora a estimulação destes nervos evoque a contração dos músculos referidos acima e as respostas, quaisquer uma delas, possam ser usadas como indexadoras da função das raízes sacrais e/ou do cone medular, vale lembrar que nem sempre o comprometimento se expressa de forma semelhante em todos os músculos. Apesar da base destes reflexos ser similar à dos reflexos flexores, possivelmente estas conexões desempenham diferentes papéis em diferentes atividades fisiológicas ${ }^{3}$. Desta forma, podemos esperar que respostas reflexas do esfíncter anal sejam importantes para os me-

Disciplinas de Urologia e Neurologia da Universidade Federal de São Paulo São Paulo, Brasil. (UNIFESP/EPM): ${ }^{1}$ Médico, Pós-graduando da Disciplina de Urologia da UNIFESP-EPM; ${ }^{2}$ Médico, Professor Afiliado, Setor de Neurofisiologia Clínica, Disciplina de Neurologia da UNIFESP-EPM; ${ }^{3}$ Médico, Livre docente, Professor Adjunto, Disciplina de Urologia da UNIFESP-EPM; ${ }^{4}$ Biomédica, Setor de Neurofisiologia Clínica, Disciplina de Neurologia da UNIFESP-EPM; ${ }^{5}$ Médico, Livre docente, Professor Titular, Disciplina de Urologia da UNIFESP-EPM; ${ }^{6}$ Médico, Livre docente, Professor Adjunto, Setor de Neurofisiologia Clínica, Disciplina de Neurologia da UNIFESP-EPM.

Recebido 23 Janeiro 2004, recebido na forma final 1 Abril 2004. Aceito 24 Maio 2004.

Dr. Gilberto Mastrocola Manzano - Rua Dr. Thirso Martins 264/52 - 04120-050 São Paulo SP - Brasil. E-mail: gmmanzano@uol.com.br 
canismos de continência fecal, enquanto que essas respostas observadas nos músculos isquio-cavernosos possivelmente sejam relevantes na manutenção do engurgitamento dos corpos cavernosos ${ }^{1}$.

A incontinência urinária feminina é hoje um importante problema de saúde pública, considerandose estimativas sugerindo que $25 \%$ da população feminina adulta sofra deste transtorno. Além do mais, estes dados tendem a crescer à medida que a média da idade populacional aumenta ${ }^{4}$. Apesar de vários tratamentos terem sido propostos, a falta de esclarecimento dos mecanismos envolvidos contribui para que o resultado do tratamento proposto nem sempre obtenha o sucesso desejado. Os mecanismos relacionados com a incontinência urinária de esforço não estão esclarecidos, tendo sido postulada uma base neural para esta condição ${ }^{5}$, embora outras causas também devam estar implicadas ${ }^{4,6}$. O reflexo pudendo-anal em mulheres é uma resposta muscular do esfíncter externo do ânus obtida pela estimulação do clitóris e, embora as vias envolvidas especificamente nesta resposta possam não estar em sua totalidade relacionadas ao mecanismo de continência urinária, foi observado que anormalidades nesta resposta podem ocorrer em muIheres incontinentes ${ }^{7}$. A obtenção e as características deste reflexo, entretanto, apresentam alguns problemas. Alguns autores acreditam que isso se deva, principalmente, às dificuldades técnicas para a estimulação do nervo pudendo em mulheres ${ }^{8}$. Além do mais, a falta de uma padronização do método, bem como a influência de variáveis como idade, dimensão corpórea e história obstétrica na la-
Tabela 1. Faixas etárias das pacientes estudadas.

\begin{tabular}{cc}
\hline Idade (anos) & $\mathrm{N}^{\circ}$ de voluntárias \\
\hline $18-30$ anos & 7 \\
$31-50$ anos & 20 \\
$>50$ anos & 4 \\
Total & 31 \\
\hline
\end{tabular}

tência deste reflexo ainda não foram totalmente esclarecidos.

Como parte de um projeto iniciado para avaliação deste problema, estudamos o reflexo pudendo-anal em uma amostra de mulheres sem queixas de incontinência urinária, tendo como objetivo a familiarização da técnica e a obtenção de valores normais preliminares. $O$ presente trabalho tem o propósito de descrever nossas observações.

\section{MÉTODO}

Realizou-se estudo prospectivo em 31 voluntárias selecionadas entre mulheres que procuraram o serviço de triagem e o ambulatório de Uroneurologia e Urologia Feminina, da Disciplina de Urologia da UNIFESP/EPM, entre janeiro de 1999 e janeiro de 2001. Após a aprovação do projeto de pesquisa pela Comissão de Ética em Pesquisa da UNIFESP e seguindo as normas estabelecidas por esta comissão, as pacientes foram orientadas a ler a Carta de Informação à Paciente (ou o Termo de Consentimento livre e esclarecido) antes do procedimento. Elas assinaram a autorização após estarem de acordo com os termos da pesquisa.

Foram considerados como critérios de inclusão pacientes que, durante o interrogatório clínico, não refe-

Tabela 2. Características da amostra em relação à idade, peso corpóreo, altura e paridade.

\begin{tabular}{lccccc}
\hline & $\begin{array}{c}\text { Idade } \\
\text { (anos) }\end{array}$ & $\begin{array}{c}\text { Peso } \\
(\mathrm{Kg})\end{array}$ & $\begin{array}{c}\text { Altura } \\
(\mathrm{m})\end{array}$ & Parto vaginal & Total \\
\hline Média & 39,48 & 61,81 & 1,58 & 0,87 & 1,55 \\
Desvio padrão & 11,47 & 8,24 & 0,08 & 1,18 & 1,15 \\
Intervalo & $20-66$ & $49-89$ & $1,44-1,78$ & $0-4$ & $0-4$ \\
Mediana & 37,00 & 60,00 & 1,58 & 0,00 & 2,00 \\
Amostra $(\mathrm{n})$ & 31 & 31 & 31 & 27 & 48 \\
\hline
\end{tabular}

Tabela 3. Média das latências (ms) do reflexo pudendo-anal.

\begin{tabular}{lcccc}
\hline & \multicolumn{2}{c}{ Estímulo $\mathrm{E}$} & \multicolumn{2}{c}{ Estímulo D } \\
& Registro $\mathrm{D}$ & Registro $\mathrm{E}$ & Registro $\mathrm{D}$ & Registro $\mathrm{E}$ \\
\hline Amostra $(\mathrm{n})$ & 30 & 30 & 31 & 31 \\
Média & 35,88 & 36,44 & 36,35 & 36,28 \\
Desvio-padrão & 4,68 & 4,45 & 6,37 & 6,23 \\
\hline
\end{tabular}

$D$, direito; $E$, esquerdo.Estímulo $(F(1,29)=0,00 ; p<0,97)$. Registro $(F(1,29)=1,30 ; p<0,26)$.

Estímulo X Registro $(F(1,29)=1,36 ; p<0,25)$. 
Tabela 4. Média das latências do reflexo pudendo-anal (ms) em relação à presença de partos vaginais.

\section{Latência}

\begin{tabular}{lcc} 
& s/ parto & c/ parto \\
\hline Amostra & 16 & 13 \\
Média & 37,16 & 35,25 \\
Desvio-padrão & 4,69 & 4,83 \\
\hline
\end{tabular}

$\mathrm{t}=0,63 ; \mathrm{p}=0,29 ; \mathrm{gl}=27$.

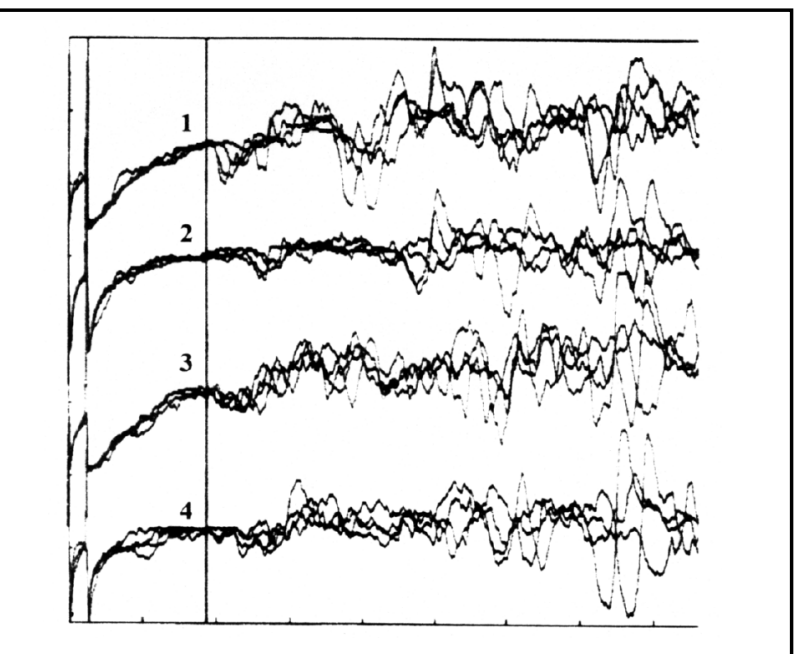

Fig 1. Registro do reflexo pudendo-anal. 1 e 2 são respostas obtidas do lado direito e esquerdo do esfincter anal após estimulação paraclitoriana direita; 3 e 4 representam respostas obtidas do lado direito e esquerdo do esfincter anal, após estimulação paraclitoriana esquerda. $100 \mu \mathrm{V} /$ div. e $20 \mathrm{~ms} /$ div.

riam perdas de urina ou fezes e no exame físico não apresentavam prolapsos genitais significativos.

Foram excluídas do estudo aquelas com história de cirurgias pélvicas ou vaginais, exceto as submetidas a parto cesariana, laqueadura tubária e cirurgia anexial unilateral. Os critérios de exclusão abrangiam também portadoras de diabetes mellitus, insuficiência renal, alcoolismo crônico, afecções neurológicas focais ou difusas, cistite intersticial, vigência de infecção urinária, disfunções miccionais (urgência sensitiva, bexiga hiperativa), além de gravidez suspeita ou confirmada.

A Tabela 1 mostra a distribuição das voluntárias segundo faixas etárias.

A idade, peso corpóreo, altura e paridade das constituintes da amostra estão demonstradas na Tabela 2. O peso corpóreo e altura foram utilizados para o cálculo do índice de massa corpórea $\left.(I M C=\text { peso/(altura })^{2}\right)$.

Utilizou-se aparelho de eletroneuromiografia NihonKohden, modelo Neuropack sigma $(\Sigma)$ de quatro canais.

As voluntárias foram colocadas em posição de litotomia. Fixou-se eletrodo terra à coxa direita. Após limpeza local com álcool a $95 \%$, eletrodos descartáveis de superfície foram aderidos na transição cutâneo-mucosa
Tabela 5. Média das latências do reflexo pudendo-anal (ms) em relação à paridade.

Latência

\begin{tabular}{lcc} 
& Nulípara & c/ parto \\
\hline Amostra & 7 & 24 \\
Média & 35,74 & 36,45 \\
Desvio-padrão & 3,18 & 4,99 \\
\hline $\mathrm{t}=0,35 ; \mathrm{p}=0,72 ; \mathrm{gl}=29$. & &
\end{tabular}

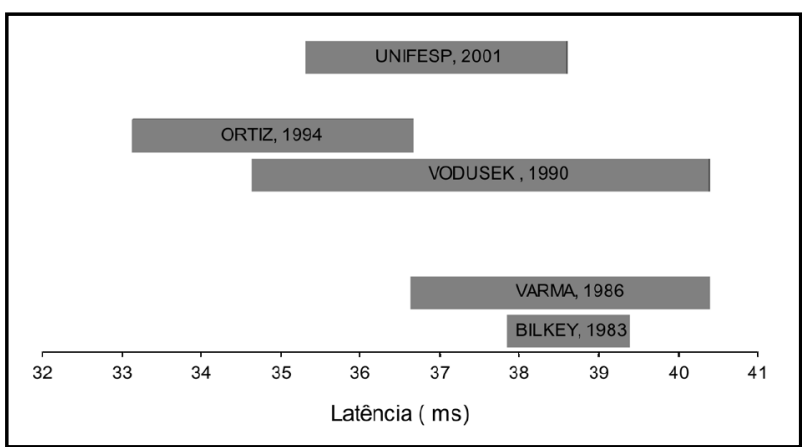

Fig 2. Latência do reflexo pudendo-anal em diferentes estudos. Os extremos laterais de cada barra representam os valores das médias acrescidos (à direita) e diminuidos (à esquerda) de dois erros padrão.

do ânus (linha pectínea) para o registro dos reflexos. Os eletrodos ativos de cada lado localizaram-se em posições equivalentes às três e nove horas. $O$ eletrodo referência foi único para ambos os lados e localizado em posição equivalente às seis horas, no sulco interglúteo.

Para a estimulação foi utilizado eletrodo bipolar, cuja distância fixa entre os pólos é de 2,0 centímetros (centro a centro). $O$ estímulo, apresentado a uma freqüência irregular sempre menor que $0,5 \mathrm{~Hz}$, foi constituído por dois pulsos retangulares de $0,2 \mathrm{~ms}$ de duração e intervalo de $5 \mathrm{~ms}$ entre os mesmos. 0 cátodo foi colocado em contato com a região paraclitoriana e o ânodo sobre a região púbica, afastado a um ângulo de $45^{\circ}$ do eixo principal da vulva. Limpeza local e colocação de gel condutor na extremidade dos eletrodos, antes do estímulo, foram realizados de rotina. Pesquisou-se de cada lado, inicialmente, o limiar sensitivo através do método de limites. A intensidade dos pulsos foi igual a três e quatro vezes o limiar sensitivo, além de intensidades absolutas de 15, 20 e até $25 \mathrm{~mA}$, obtendo-se um mínimo de quatro respostas para cada intensidade de estímulo. As respostas foram registradas utilizando-se filtros entre 20 $\mathrm{Hz}$ e $10 \mathrm{kHz}$, sensibilidade de 50 a $200 \mu \mathrm{V} /$ div. e uma base de tempo de $200 \mathrm{~ms}$ ( $20 \mathrm{~ms} / \mathrm{div}$.). Dois canais independentes registraram as respostas, através dos pares de eletrodos localizados nas posições 9-6 horas (direito) e 3-6 horas (esquerdo). A latência foi definida após estimulação de cada lado, registrando-se duas respostas: ipsilateral e contralateral. Com isso, quatro valores da latência do 
reflexo pudendo-anal foram registrados para cada intensidade do estímulo, seguindo metodologia previamente descrita9.

Como critério de leitura para se obter o valor da latência, os registros de mesma intensidade foram superpostos, determinando-se o instante do início da primeira atividade elétrica reflexa. $O$ valor da latência foi obtido para a máxima intensidade do estímulo utilizado (20 ou $25 \mathrm{~mA}$ ), visto que a resposta não aumentou significativamente de latência acima de 4 limiares de acordo com observações prévias ${ }^{10}$.

Para a comparação entre as latências obtidas do reflexo pudendo-anal foi utilizada a análise de variância (ANOVA) para medidas repetidas. Além disso, foi investigada a existência de correlação das latências com a idade e o IMC, calculando-se o coeficiente $r$ de Pearson. Para a comparação das latências entre os subgrupos com e sem a presença de parto vaginal e paridade foi utilizado o teste $t$ de student para amostras não-pareadas. Em todos os testes estatísticos, adotou-se o nível de significância de $5 \%(\alpha=0,05)$.

\section{RESULTADOS}

Respostas bilaterais foram obtidas em todas as pacientes após a estimulação de ambos os lados do clitóris (Fig 1), exceto por uma na qual não se identificou a resposta após a estimulação de um dos lados. Apesar do músculo registrado apresentar atividade tônica em repouso, em cerca de $12 \%$ das respostas uma baixa relação sinal/ruído acrescentou alguma imprecisão à determinação das latências.

A média dos valores das latências e os respectivos desvios padrão estão descritos na Tabela 3. Não se detectou diferença nas latências após os estímulos realizados à direita e à esquerda, entre os registros obtidos de cada lado e entre os 4 valores de latência obtidos para cada paciente, excetuando-se aquela que não apresentou resposta após estimulação de um dos lados. $O$ estudo da correlação entre as médias das latências de cada paciente com idade $(r=0,15 ; p<0,41)$ e IMC $(r=-0,16 ; p<0,39)$ também não evidenciou valores significantes.

Com relação a história obstétrica, as pacientes foram divididas, inicialmente, conforme a presença ou não de partos vaginais. Em seguida, as mesmas foram reagrupadas, de acordo com a paridade total de cada uma, em nulíparas e mulheres com história pregressa de partos (vaginal e cesárea).

Nesta análise também foi utilizada a média das quatro respostas de cada paciente. As Tabelas $4 \mathrm{e}$ 5 apresentam as médias dos valores da latência do reflexo pudendo-anal, conforme a distribuição das pacientes em relação à presença de parto vaginal e paridade, respectivamente. Os resultados de- monstraram não haver diferença significante entre as mesmas.

A Figura 2 apresenta um sumário dos dados retirados de estudos descritivos semelhantes ou grupos controle de trabalhos comparativos publicados, alguns dos quais tendo que incluir casuística de indivíduos masculinos, pela não separação dos mesmos por parte dos autores, o que reflete também a carência da literatura de trabalhos envolvendo o estudo desse reflexo em mulheres. Os critérios de leitura da latência foram os mesmos do presente trabalho (início da atividade reflexa), excluindo-se aqueles que utilizaram o pico da resposta como ponto para determinação da latência das respostas. Os resultados de cada trabalho são mostrados como barras flutuantes, onde a distância entre os extremos laterais representa os valores da média aritmética de cada estudo acrescido e subtraído de dois erros padrão correspondentes.

\section{DISCUSSÃO}

Poucos estudos têm enfatizado a repercussão da idade e dimensão corpórea nos resultados dos testes neurofisiológicos do assoalho pélvico. As evidências disponíveis sugerem a não existência desta influência no reflexo pudendo-anal ${ }^{11,12}$, o que está de acordo com nossas observações. Os dados do presente estudo sugerem ainda que a latência das respostas não se relaciona de forma significante ao IMC. A repercussão imediata do parto vaginal no prolongamento da resposta motora do nervo pudendo foi demonstrada em 128 gestantes antes e após o parto, tanto em primíparas quanto em multíparas. A diferença encontrada na latência foi maior logo após a primeira gestação. Alterações neurofisiológicas em nove mulheres submetidas a parto cesariana, indicada durante o trabalho de parto, também foram encontradas. A cesárea eletiva, indicada em sete casos, não apresentou diferença com o grupo controle ${ }^{13}$. A avaliação de 96 mulheres antes e no pós-parto precoce também evidenciou alterações eletromiográficas sugestivas de denervação, mesmo na primeira gestação. Evidências de re-inervação das estruturas do assoalho pélvico observadas dois meses após o parto ocorreram em $80 \%$ dos $\operatorname{casos}^{14}$. Na opinião destes autores, a ocorrência de apenas um parto vaginal já seria capaz de causar alterações neurogênicas imediatas no assoalho pélvico ${ }^{13,14}$.

Os resultados obtidos no presente estudo não evidenciaram diferenças entre os grupos com e sem partos vaginais e entre nulíparas e mulheres 
com história pregressa de partos (vaginal ou cesariana). É provável que a ocorrência de parto vaginal possa ocasionar alterações neurogênicas transitórias na musculatura pélvica. Entretanto, outros fatores precisam estar associados como multiparidade, uso de fórceps, prolongamento do período expulsivo do trabalho de parto, rotura perineal significativa e macrossomia fetal para que o comprometimento da inervação pudenda seja permanente, causando anormalidades definitivas nos testes neurofisiológicos e repercussão clínica no trato urinário baixo ${ }^{15}$.

O reflexo pudendo-anal representa uma avaliação da inervação aferente e eferente somáticas do assoalho pélvico, constituída pelo nervo pudendo. Dois componentes, denominados de precoce e tardio, podem ser identificados. O mecanismo pelo qual esses dois componentes são produzidos permanece controverso ${ }^{16,17}$. Para a obtenção desse reflexo, determina-se, inicialmente, o limiar sensitivo. A intensidade do estímulo utilizada para a definição da latência correspondendo a múltiplos do limiar tem sido descrita. Não se conseguindo uma resposta demonstrável, aumenta-se a intensidade do estímulo até obtê-lo ou gerar um desconforto significativo para a paciente. $O$ aumento progressivo da intensidade seria importante para a tolerância à realização do exame ${ }^{18}$.

O reflexo pudendo-anal apresentou-se como um teste bem reproduzível, reforçando relatos anteriores. Não se conseguiu determinar a latência de um lado em apenas uma paciente. Esses dados contrariaram afirmações publicadas previamente que sugerem que essa resposta reflexa seria difícil de registrar em um número considerável de casos $^{8}$. Porém, persiste o fato que isto possa ocorrer, sugerindo que o estudo dessa resposta apresente limitações no contexto clínico.

O limiar sensitivo foi determinado, conforme descrito, porém, a intensidade do estimulo com os valores absolutos de 15, 20 ou, em alguns casos, 25 $\mathrm{mA}$ de intensidade para determinação da latência permite concluir que, a não obtenção de resposta até a intensidade de $25 \mathrm{~mA}$ pode significar ausência do reflexo pudendo-anal. A realização do exame não trouxe desconforto considerável às voluntárias.

Não foi possível identificar os dois componentes do reflexo em todos os casos. Isso pode ter ocorrido pela utilização de eletrodos de superfície, os quais apresentam uma menor relação sinal-ruído quando comparados com os eletrodos de agulha. Entretan- to, o uso de eletrodos de superfície tem a vantagem de, além de não serem invasivos, abrangerem uma maior área do músculo ${ }^{11}$. A incerteza introduzida em aproximadamente $12 \%$ dos registros para determinação das latências sugere que a utilização de técnicas de promediação possam ser úteis no estudo destas respostas, uma vez que a variabilidade de latência do primeiro componente parece não ter impedido a utilização desta técnica com sucesso anteriormente ${ }^{11}$.

Foi relatado previamente não haver diferença entre as respostas obtidas em ambos os lados para os valores da latência do reflexo pudendo-anal ${ }^{14}$ o que foi corroborado no presente trabalho. 0 contraste com os dados da literatura ${ }^{7,8,12,19}$ demonstram uma semelhança dos resultados obtidos com a maioria das publicações. Isso pode representar uma uniformidade da metodologia, dos critérios de leitura e das diferentes populações estudadas.

\section{REFERÊNCIAS}

1. Uher E-M, Swash M. Sacral reflexes physiology and clinical application. Dis Colon Rectum 1998;41:1165-1177.

2. Persen E, Harving H, Klemar B, Torring J. Human anal reflexes. J Neurol Neurosurg Psychiatry 1978;9:813-818.

3. Vereecken RL, de Meirsman J, Puers B, van Mulders J. Electrophysiological exploration of the sacral conus. J Neurol Neurosurg Psychiatry 1982;45:135-144.

4. Jarvis GF. Stress Incontinence. In Mundy Stephenson, Wein. Urodinamics: principles, practice and application, 2nd ed. Edinburg: Churchill Livingstone, 1994;299-326.

5. Swash M. New concepts in incontinence. Br Med J 1985;290:4-5.

6. Warrell DW. Pathophysiology of genuine stress incontinence. In Drife, Hilton, Stanton. Micturition. London: Springer-Verlag, 1989:203-208.

7. Varma JS, Fidas A, McInnes A, Smith AN, Chisholm GD. Neurophysilogical abnormalities in genuine female stress urinary incontinence. $\mathrm{Br}$ J Obstet Gynaecol 1988;95:705-710.

8. Vodusek DB. Pudendal SEP and bulbocavernosus reflex in women. Electroenceph Clin Neurophysiol 1990;77:134-136.

9. Benson JT. Pelvic floor neurophysiology. In American Association of Electrodiagnostic Medicine. Workshop, 1998

10. Cavalcanti GA, Manzano GM, Giuliano L, Nóbrega JAM, Bruschini H. Reflexo clítoro-anal em mulheres normais: respostas a variações de intensidade de estimulo. Anais do XXVII Congresso Brasileiro de Urologia. Rio de Janeiro, 1999;137:T537.

11. Varma JS, Smith AN, McInnes A. Electrophysiological observations on the human pudendo-anal reflex. J Neurol Neurosurg Psychiatry 1986;49:1411-1416.

12. Bilkey WJ, Awad EA, Smith AD. Clinical application of sacral reflex latency. J Urol 1983;129:1187-1189.

13. Sultan AH, Kamm MA, Hudson CN. Pudendal nerve damage during labour: prospective study before and after childbirth. Br J Obstet 1994;101:22-28.

14. Allen RE, Hosker GL, Smith ARB, Warrell DW. Pelvic floor damage and childbirth: a neurophysiological study. Br J Obstet Gynaecol 1990;97:770-779.

15. Wall LL. The muscles of the pelvic floor. Clin Obstet Gynecol 1993;36: 910-925.

16. Krane RJ, Siroky MB. Studies on sacral-evoked potentials. J Urol 1980;124:872-876

17. Dick HC, Bradley WE, Scott FB, Timm GW. Pudendal sexual reflexes: electrophysiological investigations. Urology 1974;3:376-379.

18. Galloway NTM, Chisholm GD, McInnes A. Patterns and significance of the sacral evoked response (the urologist's knee jerk). Br J Urol 1985;57:145-147.

19. Ortiz OC, Bertotti AC, Nuñez JDR. Pudendal reflexes in women with pelvic floor disorders. Zentralbl Gynakol 1994;116:561-565. 